\title{
Efficiency and Reliability Analysis of Self-Adaptive Two-Stage Fuzzy Control System in Complex Traffic Environment
}

\author{
Mingzhi Wang, ${ }^{1}$ Xianyu Wu ${ }^{D},{ }^{2}$ He Tian, ${ }^{1}$ Jie Lin, ${ }^{2}$ Meimei He, ${ }^{2}$ and Liuqing Ding ${ }^{2}$ \\ ${ }^{1}$ Laboratory for Traffic and Transport Planning Digitalization, Transport Planning and Research Institute Ministry of Transport, \\ Beijing, China \\ ${ }^{2}$ Key Laboratory of Transport Industry of Big Data Application Technologies for Comprehensive Transport, \\ Beijing Jiaotong University, Beijing, China \\ Correspondence should be addressed to Xianyu Wu; wuxy@bjtu.edu.cn
}

Received 30 November 2021; Revised 24 December 2021; Accepted 11 January 2022; Published 27 January 2022

Academic Editor: Wenxiang Li

Copyright ( 2022 Mingzhi Wang et al. This is an open access article distributed under the Creative Commons Attribution License, which permits unrestricted use, distribution, and reproduction in any medium, provided the original work is properly cited.

\begin{abstract}
In this paper, a self-adaptive, two-stage fuzzy controller is established to realize the real-time online optimization of traffic signal timing plan, which takes multimodels of transportation as the research object to analyze the reliability of the control system at the isolated urban intersection. In this system, the first stage calculates traffic urgency degree for all red phases and selects the red phase with maximum traffic urgency degree as the next green phase. The second stage determines whether to extend or terminate the current signal phase. Aiming at the problems of the parameters of membership functions empirical settings and insufficient response to the real-time fluctuation in traffic flow, the controller introduces an improved hybrid genetic algorithm to solve it and enable the controller to self-learn. Finally, a microsimulation platform is constructed based on the VISSIM and Python language to evaluate the efficiency and reliability of the controller under complex actual traffic conditions. Results showed that the average delay time per vehicle is reduced by $14.59 \%$, while the average number of stops per vehicle is reduced by $0.71 \%$ compared with the traditional control method. Results indicate that the traffic signal timing plan generated by the controller can efficiently improve the intersection traffic capacity and has good efficiency and reliability under the condition of medium saturation and unsteady flow.
\end{abstract}

\section{Introduction}

At present, with the development of shared mobility and traffic detection techniques theories, many scholars in the field of urban traffic engineering are paying more attention to the collaborative optimization of multi-transport models in traffic safety [1-3], traffic operation efficiency and reliability $[4,5]$, and environmental protection [6-8]. Driven by the rapid development of the mobile Internet and the sharing economy, dockless bike-sharing systems have grown in popularity in China [9]. For example, in Beijing, the total daily average of cycling trips has reached 5.6 million by 2020 , up from 4.62 million in 2017, surpassing the trips by bus and subway and becoming the third-largest way of travel after walking and car [10]. In addition, in the face of congested traffic, people are more likely to choose to use dockless bike-sharing to complete short-distance travel
[11]. Shared mobility has led to more walking, and cycling trips significantly changed intersections' traffic flow structure and brought new challenges to the intersection signal timing optimization. Therefore, considering the travel needs of bicycles and pedestrians in the process of signal timing optimization has become an important research content. At the same time, with the emergence of new detection technologies such as microwave radar and high point video, it is possible to analyze the efficiency and reliability of signal timing control systems in the real environment.

In some developing countries, such as China and India, the most signalized intersection' control mode is fixed-time or actuated control due to complex traffic flow and lack of effective observation data [12]. As the fixed-time control cannot respond to the real-time change of traffic demand, vehicle actuated control is better than fixed-time control. 
Though it can optimize the timing scheme of signalized intersections according to the real-time traffic demand, the setting of critical parameters relies on urban traffic engineers' personal experience [13]. In the past few decades, to address these deficiencies, a variety of adaptive signal control systems have been proposed by Gartener [14], Lin and Cooke [15], Kronborg and Davidson [16], and Lin and Vijayakumar [17]. Because of its excellent real-time and robustness, fuzzy control has become one of the most common intelligent control methods.

Fuzzy control was introduced into the traffic control field in 1977 by Pappis and Mamdani [18]. Since then, single-level, two-level, and three-level fuzzy controllers have been proposed one after another [19-22]. However, as the parameters are pre-defined by experts and unable to change, this fuzzy controller is non-responsive to the realtime fluctuation of traffic demand and disability to learn [23]. To solve the problems above, Henry et al. [24], Bingham [25], Rida et al. [26], and Xu et al. [27] introduce heuristic algorithm, such as reinforcement learning, neural network, and ant colony to optimize fuzzy controller parameters according to the historical traffic data. The simulation result indicates this adaptive fuzzy controller is more effective than a traditional fuzzy controller, but the performance of these traffic signal control methods needed large and valid sample sets. Genetic algorithm (GA) has been widely used with the ability of global search and does not depend on experiential knowledge and a large amount of historical traffic flow data $[22,28]$. In light of this, Liu and Zuo [29] take a four-phase isolated signalized intersection as a research object and uses a genetic algorithm to optimize fuzzy rules and membership functions of the fuzzy control. Shahsavari et al. [30] take the total length of traffic queue and pedestrian queue in all phase of isolated intersection as optimization goals, construct a fuzzy multiobjective optimization model, and use a genetic algorithm to optimize the cycle time of the traffic light systems. Yu et al. [31] established a multi-objective optimization model with traffic capacity, average parking delay, and exhaust emissions as optimization goals and used genetic algorithms to optimize the signal cycle time and effectively green light time. However, in the above study, the traffic flow distribution is generally assumed to obey a particular traffic arrival distribution, which does not reflect the fluctuation of actual traffic demand. In addition, pedestrians and non-motor vehicles are also almost not taken into account. Except that, the simulation experiment designed in the existing research has the disability to reflect actual vehicle driving behavior and mixed traffic flow characteristics.

The aim of this paper was to take the travel needs of nonmotor vehicles, pedestrians, and motor vehicles into account during the optimization process of signal timing scheme. In addition, this paper introduces a hybrid genetic algorithm to make the signal control strategy have the ability of autonomous learning. At the same time, to verify the efficiency and reliability of this control system, the simulation parameters of the platform are calibrated according to the actual vehicle trajectory data.

\section{Research Scope and Model Structure}

2.1. Experimental Test Intersection. The plan of the signalized isolated intersection is shown in Figure 1. As the intersection has through-left movement, this paper selects a split-phase to control the intersection. Since there is a dedicated rightturn lane at this intersection, right-turn traffic flow is not considered in the research.

As shown in Figure 1, each approach has one microwave radar and many cameras to detect the vehicles' status information of each lane, such as cross ID, latitude, and longitude, with an average sampling interval of 80 milliseconds. According to the trajectory data, vehicles' temporal and spatial distribution characteristics can be obtained [32], making it possible to get the real intersection traffic flow information and analyze the driving characteristics of drivers. The daily file size of high-precision track data used in the research is about $1 \mathrm{G}$, and about 8 million track point records are generated. The primary data attributes are shown in Table 1.

Due to the complexity of the real environment, possible packet loss during data transmission, buffer data overflow and on-board equipment and roadside unit failure, there will be problems in the collection of high-precision track data, such as missing field values, missing track information, and change of track ID. At the same time, roadside parking, bad weather, or temporary traffic control may also cause the obtained high-precision trajectory data to fail to reflect the normal operation state of vehicles at the intersection, so the data need to be preprocessed before use. Data cleaning work has been done in a previous work [33], and hence the details are not included to avoid redundancy.

\subsection{Self-Adaptive Two-Stage Fuzzy Control Algorithm.} The primary process of the self-adaptive two-stage fuzzy control algorithm is as follows: (1) set a minimum green time to a phase until the remainder of the green time is $1 \mathrm{~s}$; (2) calculates the red urgency and green urgency of different approaches according to the real-time motor vehicle, nonmotor vehicle, and pedestrians traffic flow data; (3) select the red phase with greatest phase urgency as the next candidate phase; (4) comparing the selected red phase urgency with green phase urgency, decided whether to extend or terminate the current signal phase; (5) if the length of the green phase exceeds maximum green duration, then terminal the current signal phase, otherwise, continue; (6) according to the evaluation of the scheme, decided whether to optimize the parameters of membership functions; (7) if the simulation time does not reached, skip to (2) and continue.

2.3. Structure of Self-Adaptive Two-Stage Fuzzy Controller. Figure 2 shows the structure of the self-adaptive two-stage fuzzy controller. As we can see from the figure, the controller has two main components: (1) two-stage fuzzy controller, which includes four parts: red urgency judgment module, pedestrian urgency judgment module, green urgency judgment module, and decision module; (2) parameter optimization module based on improved hybrid genetic algorithm. 


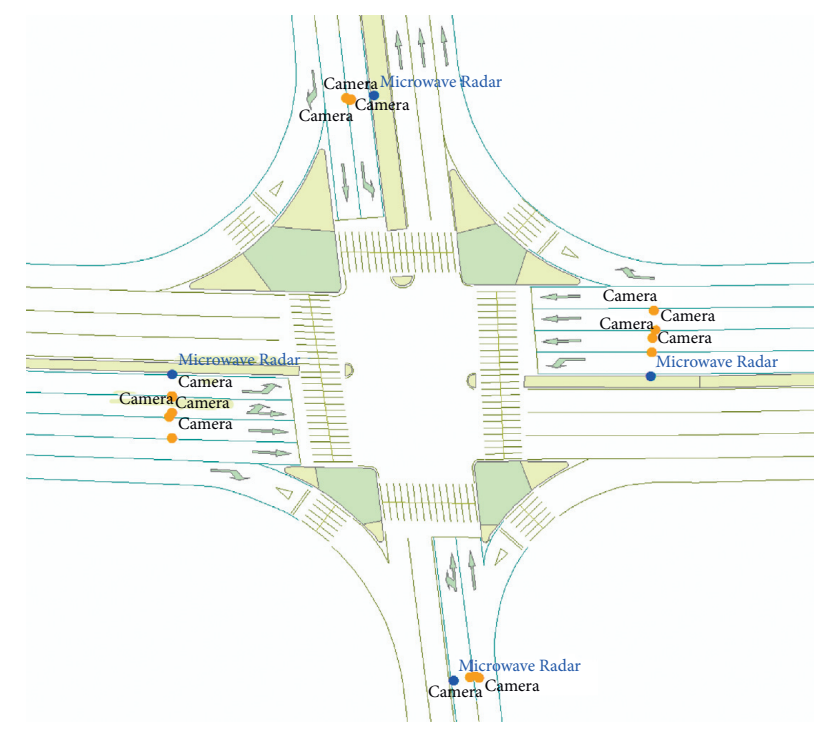

Figure 1: Intersection plan.

TABLE 1: Introduction of the source data.

\begin{tabular}{lc}
\hline Field name & Attribute description \\
\hline Time & Unix timestamp, in millisecond \\
crossID & Intersection ID \\
Sequence & Track serial number \\
trackID & Track ID, unique for a short time \\
entryRoad & Entry road direction \\
exitRoad & Exit road direction \\
objectType & Object type: 0 unknown, 1 motor \\
vehicleType & Vehicle type: 1 car, 2 truck, etc \\
plateNumber & Plate number \\
$\mathrm{N}$ & EPSG:32650-WGS 84/UTM \\
$\mathrm{E}$ & EPSG:32650-WGS 84/UTM \\
$\mathrm{Z}$ & EPSG:32650-WGS 84/UTM \\
\hline
\end{tabular}

2.4. Computation of the Queue Lengths. When the phase is red, the queue lengths in the approach direction $i$ are expressed as

$$
Q_{i}=Q_{r i}+\sum_{j=1}^{t} q_{i j}
$$

where $Q_{i}$ is the queue lengths of an approach direction $i ; Q_{r i}$ is the number of remaining vehicles when the phase is switched to red phase; $t$ is the duration of the red phase of approach direction $i$; and $q_{i j}$ is the amount of arriving vehicles of approach direction $i$ duration interval.

When the phase is green, the queue lengths in the approach direction $i$ are expressed as

$$
Q_{i}=Q_{g i}+\sum_{j=1}^{t} q_{i j}-\sum_{j=1}^{t} q_{i j}^{\prime},
$$

where $q_{i j}^{\prime}$ is the number of vehicles through stop-line of approach direction $i$ duration interval and $Q_{g i}$ is the number of remaining vehicles when switched to the green phase.

\section{Design of Self-Adaptive Two-Stage Fuzzy Control}

3.1. First Stage. Since the first stage has three fuzzy inputs, theoretically, the number of rules necessary for gathering all the possible input combinations for the three-term fuzzy controller is $5 \times 5 \times 5=125$. In practice, the design of a fuzzy controller with so many fuzzy control rules will occupy a large amount of memory and long processing time [34, 35]. Therefore, this study divided the first stage into three submodules modules, namely: red urgency judgment module, pedestrian urgency judgment module, and green urgency judgment module, each with two fuzzy inputs and one output variable.

In these sub-modules, phase urgency on each approach direction is determined by the maximum red/green urgency and pedestrian urgency. For example, if approach direction $i$ is in red and its red urgency is 2.3 , and pedestrian urgency is 2.2 , then $i$ would have a phase urgency of 2.3 and is named red urgency. The calculation process of the urgency of each submodule is shown below.

3.1.1. Red Urgency Judgment Module. In this module, the input variable is the queue length of motor vehicles $r$, the red phase duration time $t r$. The output variable is urgency $U r$. The domain of $q r$ is $(0,16)$, the linguistics are \{Very Few Vehicles, Rarely Few Vehicles, Medium Vehicles, Rarely More Vehicles, Very More Vehicles\}, short as $\{\mathrm{VFV}, \mathrm{RFV}$, $\mathrm{MV}, \mathrm{RMV}, \mathrm{VMV}\}$. The domain of $t r$ is $(0,120)$, the linguistics are VVery Short Time, Rarely Short Time, Medium Time, Rarely Long Time, Very Long Time\}, short as \{VST, RST, MT, RLT, VLT\}. The domain of $U r$ is $(0,4)$, the linguistics are \{Very Low, Low, Medium, Height, Very Height\}, short as $\{\mathrm{VL}, L, \mathrm{M}, \mathrm{H}, \mathrm{VH}\}$.

With linguistic descriptions of $q r, t r$ as input, the urgency in red direction, $U r$, is determined by the fuzzy rules given in Table 2. For example, if $t r$ is VSF, and $q r$ is VFV, then $U r$ is VL.

3.1.2. Pedestrian Urgency Judgment Module. In this module, the input variable is the traffic flow of non-motor and pedestrian on each approach direction $q p$, the phase duration time is $t p$, and the output variable is urgency $U p$. The domain of $q p$ is $(0,60)$, the linguistics are \{Very Few Pedestrians, Rarely Few Pedestrians, Medium Pedestrians, Rarely More Pedestrians, Very More Pedestrians\}, short as $\{$ VFP, RFP, MP, RMP, VMP $\}$. The domain of $t p, U p$ is $(0$, $120)$ and $(0,4)$, respectively. The linguistic subsets of $t p, U p$ are same as red urgency judgment module. The rules of these modules are shown in Table 3.

3.1.3. Green Urgency Judgment Module. In this module, the input variable is the number of remaining vehicles $q g$, and green extension time $\mathrm{tg}$. The domain and terms of each variable are shown in Table 2. The domain of $q g, t g, U g$ is $(0,16),(0,120),(0,4)$, respectively. The linguistic subsets of 


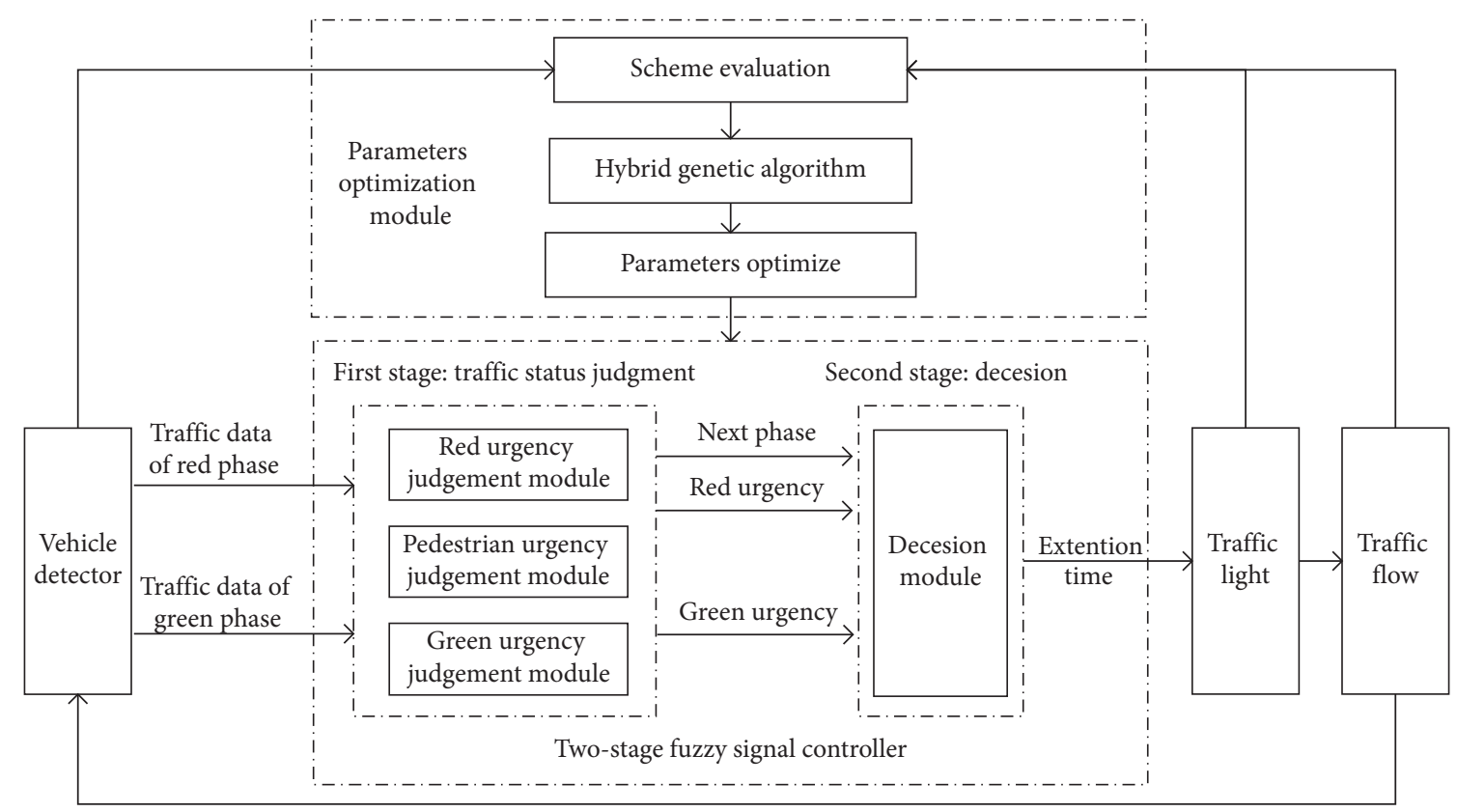

FIgURE 2: The diagram of adaptive two-stage fuzzy controller.

TABLE 2: Fuzzy rules for the red urgency judgment module.

\begin{tabular}{|c|c|c|c|c|c|c|}
\hline \multirow{2}{*}{\multicolumn{2}{|c|}{$U r$}} & \multicolumn{5}{|c|}{$q r$} \\
\hline & & VFV & RFV & MV & RMV & VMV \\
\hline \multirow{5}{*}{$t r$} & VST & VL & VL & $\mathrm{VL}$ & $\mathrm{L}$ & $\mathrm{M}$ \\
\hline & RST & VL & VL & $\mathrm{L}$ & M & $\mathrm{H}$ \\
\hline & MT & $\mathrm{L}$ & M & $\mathrm{M}$ & $\mathrm{H}$ & VH \\
\hline & RLT & M & $\mathrm{H}$ & $\mathrm{H}$ & VH & VH \\
\hline & VLT & $\mathrm{VH}$ & VH & $\mathrm{VH}$ & $\mathrm{VH}$ & VH \\
\hline
\end{tabular}

TABLE 3: Fuzzy rules for the pedestrian urgency judgment module.

\begin{tabular}{|c|c|c|c|c|c|c|}
\hline \multirow{2}{*}{\multicolumn{2}{|c|}{$U p$}} & \multicolumn{5}{|c|}{$q p$} \\
\hline & & VFP & RFP & MP & RMP & VMP \\
\hline \multirow{5}{*}{$t p$} & VST & VL & VL & VL & $\mathrm{L}$ & $\mathrm{M}$ \\
\hline & RST & VL & VL & $\mathrm{L}$ & $\mathrm{M}$ & $\mathrm{H}$ \\
\hline & MT & $\mathrm{L}$ & $\mathrm{M}$ & M & $\mathrm{H}$ & VH \\
\hline & RLT & $\mathrm{M}$ & $\mathrm{H}$ & $\mathrm{H}$ & $\mathrm{VH}$ & VH \\
\hline & VLT & $\mathrm{VH}$ & $\mathrm{VH}$ & $\mathrm{VH}$ & $\mathrm{VH}$ & $\mathrm{VH}$ \\
\hline
\end{tabular}

each variable are the same as the red urgency judgment module. The rules of these modules are shown in Table 4 .

The research uses the Gaussian fuzzy membership function to describe all parameters and uses the center of gravity method to de-fuzz output results.

$$
\begin{array}{r}
f(x, \mu, \sigma)=e^{-\left((x-\mu)^{2} / 2 \sigma^{2}\right)}, \\
Z=\frac{\int x f(x) \mathrm{d}(x)}{\int f(x) \mathrm{d}(x)},
\end{array}
$$

where $\mu$ represents the membership function center; $\sigma$ denotes the membership function width; and $x$ indicates the element; and $f(x)$ is the membership function.

\begin{tabular}{|c|c|c|c|c|c|c|}
\hline \multirow{2}{*}{\multicolumn{2}{|c|}{$U g$}} & \multicolumn{5}{|c|}{99} \\
\hline & & VFV & RFV & MV & RMV & VMV \\
\hline \multirow{5}{*}{$\operatorname{tg}$} & VST & $\mathrm{L}$ & $\mathrm{M}$ & $\mathrm{H}$ & $\mathrm{VH}$ & $\mathrm{VH}$ \\
\hline & RST & $\mathrm{L}$ & $\mathrm{L}$ & $\mathrm{M}$ & $\mathrm{H}$ & $\mathrm{VH}$ \\
\hline & MT & VL & $\mathrm{L}$ & $\mathrm{L}$ & M & $\mathrm{H}$ \\
\hline & RLT & VL & VL & $\mathrm{L}$ & $\mathrm{L}$ & $\mathrm{M}$ \\
\hline & VLT & VL & VL & VL & $\mathrm{L}$ & $\mathrm{L}$ \\
\hline
\end{tabular}

Table 4: Fuzzy rules for the green urgency judgment module.

3.2. Second Stage. The decision module takes $U r$ and $U g$ as an input variable, and the output variable $D c$ represents whether to extend the green time or deliver the right of way to the selected phase $(\operatorname{Pr})$. The domain of $D c$ is $(0,1)$. It is divided into two linguistic subsets $\{$ No, Yes $\}$, abbreviated as $\{\mathrm{N}, \mathrm{Y}\}$. The rules of these modules are shown in Table 5.

3.3. Parameter Optimization Module. To enable the two-stage fuzzy controller have autonomous learning ability, this paper uses a hybrid genetic algorithm to optimize the membership function of the fuzzy variable and uses a rolling horizon to set the parameters of these membership functions $[28,36]$. The framework of the module is shown in Figure 3. Furthermore, the process of parameter optimization is as follows: (1) Detect real-time traffic flow during the control time horizon, evaluate the scheme, and decide whether to optimize membership functions' parameters. If so, go to step 2; otherwise, end. (2) Optimize the parameters of $\mu$ in membership function by hybrid genetic algorithm (HGA) based on historical traffic flow data. (3) Update the $\mu$ parameters of the fuzzy controller in time, according to the optimized membership function. (4) Repeat the above process until the simulation time is reached. 
TABLE 5: Fuzzy rules for the decision module.

\begin{tabular}{|c|c|c|c|c|c|c|}
\hline \multicolumn{2}{|c|}{$D c$} & \multicolumn{5}{|c|}{$U r$} \\
\hline & $D c$ & VL & $\mathrm{L}$ & $\mathrm{M}$ & $\mathrm{H}$ & VH \\
\hline \multirow{5}{*}{$U g$} & $\mathrm{VL}$ & $\mathrm{Y}$ & $\mathrm{Y}$ & $\mathrm{Y}$ & $\mathrm{Y}$ & $\mathrm{Y}$ \\
\hline & $\mathrm{L}$ & $\mathrm{N}$ & $\mathrm{Y}$ & $\mathrm{Y}$ & $\mathrm{Y}$ & $\mathrm{Y}$ \\
\hline & $\mathrm{M}$ & $\mathrm{N}$ & $\mathrm{N}$ & $\mathrm{Y}$ & $\mathrm{Y}$ & $\mathrm{Y}$ \\
\hline & $\mathrm{H}$ & $\mathrm{N}$ & $\mathrm{N}$ & $\mathrm{N}$ & $\mathrm{Y}$ & $\mathrm{Y}$ \\
\hline & $\mathrm{VH}$ & $\mathrm{N}$ & $\mathrm{N}$ & $\mathrm{N}$ & $\mathrm{N}$ & $\mathrm{Y}$ \\
\hline
\end{tabular}

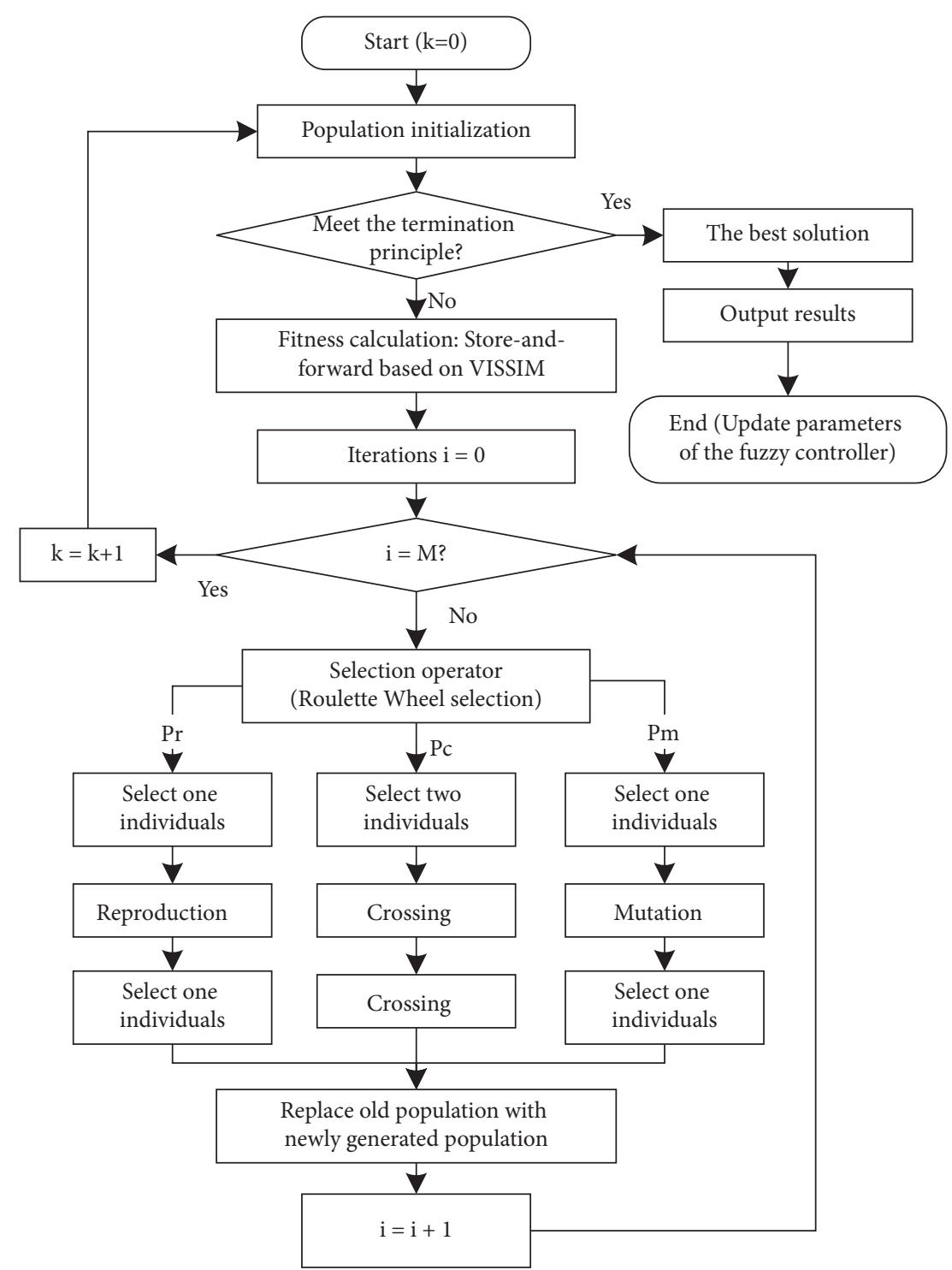

FIGURE 3: Framework of the parameter optimization module.

As shown in Figure 3, this study uses floating-point coding to encode the chromosome and sets a limited value range for each control parameter to avoid the generation gap phenomenon so that no fuzzy rules are activated after the parameters were input. For example, if the parameters of $\mu^{\prime}$ are the $k^{\text {th }}$ element in the linguistic sets of $q r$, as the domain of $q r$ is $(0,16)$, so the range of $\mu^{\prime}$ is $((k-1) *(16-0 / 5-1)-$ $2,(k-1) *(16-0 / 5-1)+2)$. The fitness value of $C$, denoted by $f(C)$, is computed by

$$
f(C)=\frac{1}{(1+d)},
$$

where $d$ is the average delay time per vehicle obtained by the simulation platform. 
The parameters of $\mathrm{Pr}, \mathrm{Pc}, \mathrm{Pm}$ are calculated by

$$
\begin{aligned}
\operatorname{Pr} & =\frac{f(i)}{\sum_{n=1}^{k} f(C)}, \\
P c & = \begin{cases}P c_{1} \times \frac{1}{\left(P c_{1}-P c_{1}\right)+N}, & N_{1} \geq N_{2}, \\
0.9, & N_{1}<N_{2},\end{cases} \\
P m & = \begin{cases}P m_{1} \times \frac{1}{\left(P m_{1}-P m_{1}\right)+N}, & N_{1} \geq N_{2}, \\
0.2, & N_{1}<N_{2},\end{cases} \\
N & =\exp \left(\frac{\left(N_{1}-N_{2}\right)}{\left(N_{3}-N_{2}\right)}\right),
\end{aligned}
$$

where $k$ is the number of individuals; $f(i)$ is fitness value of individual of $i ; \operatorname{Pr}$ is select probability; $P c$ is crossing probability; $P c_{1}$ is the minimum crossing probability, 0.2 ; $\mathrm{Pc}_{2}$ is the maximum crossing probability, 0.9; $\mathrm{Pm}$ is mutation probability; $\mathrm{Pm}_{1}$ is the minimum crossing probability, $0.01 ; \mathrm{Pm}_{2}$ is the maximum crossing probability, 0.2 ; and $N_{1}, N_{2}, N_{3}$ are the rank index of minimum, average, and maximum fitness, respectively.

\section{Efficiency and Reliability Analysis}

\subsection{Simulation Environment}

4.1.1. Structure of the Simulation Platform. The proposed control strategy was verified in a prevailing microscopic traffic simulation environment, VISSIM. The control strategy and optimization algorithm are compiled on the visual studio code platform and transmitted to the VISSIM via the COM interface. At each step, according to the current simulation information, the adaptive two-stage fuzzy controller decided whether to terminate or extend the current green signal phase and judgment whether to optimize the membership function or maintain the status quo. The structure of the VISSIM simulation platform is shown in Figure 4.

4.1.2. VISSIM Parameter Calibration. VISSIM simulation software uses a large number of independent simulation parameters to reflect real vehicle operation, traffic flow characteristics, and driving behavior. However, these parameters are calibrated according to Germany and other western countries' measured data and may not be suitable for China's actual situation. As these parameters have an important impact on the simulation results, it is necessary to calibrate the default simulation parameters according to the actual situation of the intersection before the simulation. In this study, the seven parameters that significantly impact the simulation results are calibrated, including desired speed distribution, maximum acceleration/deceleration, expected acceleration/deceleration, average standstill distance, additive

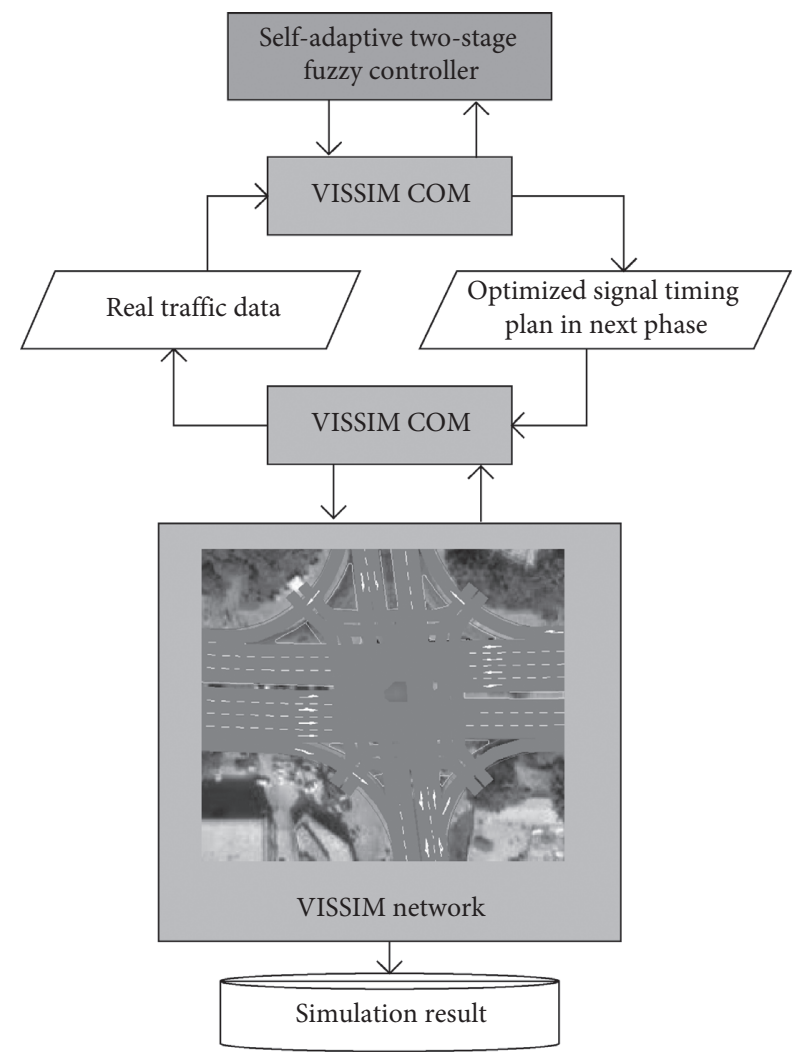

FIGURE 4: The structure of the VISSIM simulation platform.

part, and multiple parts of safety distance [37-39]. The specific calibration process can refer to the literature [33].

\subsection{Experimental Design}

4.2.1. Traffic Flow. The traffic flow data of motor vehicles at the intersection extracted based on high-precision trajectory data are shown in Table 6. Data extraction work has been done in a previous work [33], and hence the details are not included to avoid redundancy.

Since trajectory data cannot obtain the traffic flow data of non-motor vehicles and pedestrians, this study assumes that the arrival rates of non-motor vehicles and pedestrians in each approach direction obey Poisson distribution [40]:

$$
P(x)=\frac{\lambda t^{x} e^{-\lambda t}}{x !}, \quad x=0,1,2, \ldots,
$$

where $P(x)$ is the probability of $x$ non-motor or pedestrian arrival in $t$ interval; $\lambda$ is the average arrival rate per unit time, $0-0.4$; and $t$ is phase duration of current approach direction.

4.2.2. Traffic Flow Structure. The traffic flow structure data of motor vehicles at the intersection extracted based on trajectory data is shown in Table 7.

4.2.3. Simulation Parameters Calibration. The simulation parameters are calibrated based on the intersection geometry (Figure 1) and traffic flow characteristics. The parameter calibration results are shown in Table 8 . 
Table 6: Traffic flow (pcu/15 minutes).

\begin{tabular}{|c|c|c|c|c|c|c|c|c|}
\hline Time & WBL & WBT & SBL & SBT & NBT & NBL & EBT & EBL \\
\hline 0:00 & 6 & 18 & 6 & 2 & 5 & 4 & 35 & 9 \\
\hline $0: 15$ & 3 & 22 & 4 & 4 & 3 & 3 & 26 & 4 \\
\hline $0: 30$ & 9 & 14 & 1 & 2 & 2 & 3 & 16 & 6 \\
\hline$\ldots$ & $\ldots$ & $\ldots$ & $\ldots$ & $\ldots$ & $\ldots$ & $\ldots$ & $\ldots$ & $\ldots$ \\
\hline $7: 30$ & 8 & 134 & 22 & 0 & 3 & 16 & 75 & 39 \\
\hline $7: 45$ & 5 & 199 & 28 & 5 & 13 & 14 & 87 & 60 \\
\hline 8:00 & 14 & 236 & 33 & 14 & 28 & 16 & 102 & 85 \\
\hline $8: 15$ & 26 & 245 & 45 & 13 & 32 & 26 & 136 & 100 \\
\hline $8: 30$ & 12 & 206 & 49 & 16 & 31 & 16 & 130 & 132 \\
\hline $8: 45$ & 18 & 230 & 36 & 12 & 24 & 20 & 128 & 110 \\
\hline 9:00 & 15 & 181 & 38 & 17 & 10 & 11 & 130 & 54 \\
\hline $9: 15$ & 14 & 161 & 37 & 13 & 10 & 17 & 117 & 41 \\
\hline $9: 30$ & 14 & 147 & 19 & 10 & 6 & 24 & 119 & 40 \\
\hline $9: 45$ & 9 & 156 & 20 & 5 & 2 & 9 & 78 & 29 \\
\hline$\ldots$ & $\ldots$ & $\ldots$ & $\ldots$ & $\ldots$ & $\ldots$ & $\ldots$ & $\ldots$ & $\ldots$ \\
\hline 19:00 & 1 & 34 & 15 & 1 & 2 & 5 & 22 & 4 \\
\hline 19:15 & 7 & 36 & 19 & 3 & 0 & 0 & 45 & 8 \\
\hline 19:30 & 4 & 20 & 16 & 4 & 3 & 0 & 2 & 0 \\
\hline
\end{tabular}

In this table, WBL means westbound left, WBT means westbound through, and so on.

TABLE 7: Traffic flow structure.

\begin{tabular}{|c|c|c|c|c|c|}
\hline \multirow{2}{*}{ Structure } & \multicolumn{3}{|c|}{ Vehicle } & \multicolumn{2}{|c|}{ Nonmotor (\%) } \\
\hline & Car (\%) & HGV (\%) & Minibus (\%) & Nonmotor (\%) & Pedestrian (\%) \\
\hline WBL & 93.1 & 3.1 & 3.8 & 60 & 40 \\
\hline WBT & 98.1 & 1.0 & 0.9 & 60 & 40 \\
\hline SBL & 83.9 & 4.5 & 11.7 & 60 & 40 \\
\hline SBT & 85.4 & 5.5 & 9.1 & 60 & 40 \\
\hline NBL & 88.1 & 4.2 & 7.7 & 60 & 40 \\
\hline NBT & 88.1 & 4.6 & 7.3 & 60 & 40 \\
\hline EBL & 90.8 & 3.2 & 6.0 & 60 & 40 \\
\hline EBT & 83.1 & 8.5 & 8.4 & 60 & 40 \\
\hline
\end{tabular}

TABLE 8: Simulation parameters.

\begin{tabular}{lc}
\hline Parameters & Desired value \\
\hline Duration of simulation & $19.5 \mathrm{~h}$ \\
Green extension & $2 \mathrm{~s}$ \\
Maximum cycle length & $120 \mathrm{~s}$ \\
Amber time & $3 \mathrm{~s}$ \\
All red time & $2 \mathrm{~s}$ \\
Minimum duration of the WB/EB approach & $21 \mathrm{~s}$ \\
Minimum duration of the SB/NB approach & $13 \mathrm{~s}$ \\
Through capacity per lane & $1650 \mathrm{pcu} / \mathrm{h}$ \\
Left-turn/right-turn capacity per lane & $1550 \mathrm{pcu} / \mathrm{h}$ \\
Simulation runs & 20 \\
\hline
\end{tabular}

\section{Results and Discussion}

Figures 5-7 show the simulation results of fixed-time control (FTC), traditional fuzzy control (TFC), and hybrid genetic algorithm fuzzy control (HGAFC). Compared with FTC, the average delay time per vehicle of HGAFC is reduced by $14.59 \%$, and the average number of stops per vehicle is reduced by $0.71 \%$; the average delay time per vehicle of TFC is reduced by $11.08 \%$, and the average number of stops per vehicle is reduced by $0.33 \%$.
The following inferences can be drawn based on Figures 5-7:

(1) In the early morning, the effect of the control algorithm is the same. The reason is that when the traffic flow of each approach of the intersection is low, the vehicle queue length is short. The fuzzy control's main influence factor is each phase's red/ green light duration, and the fuzzy control is in a fixed-time control state. 

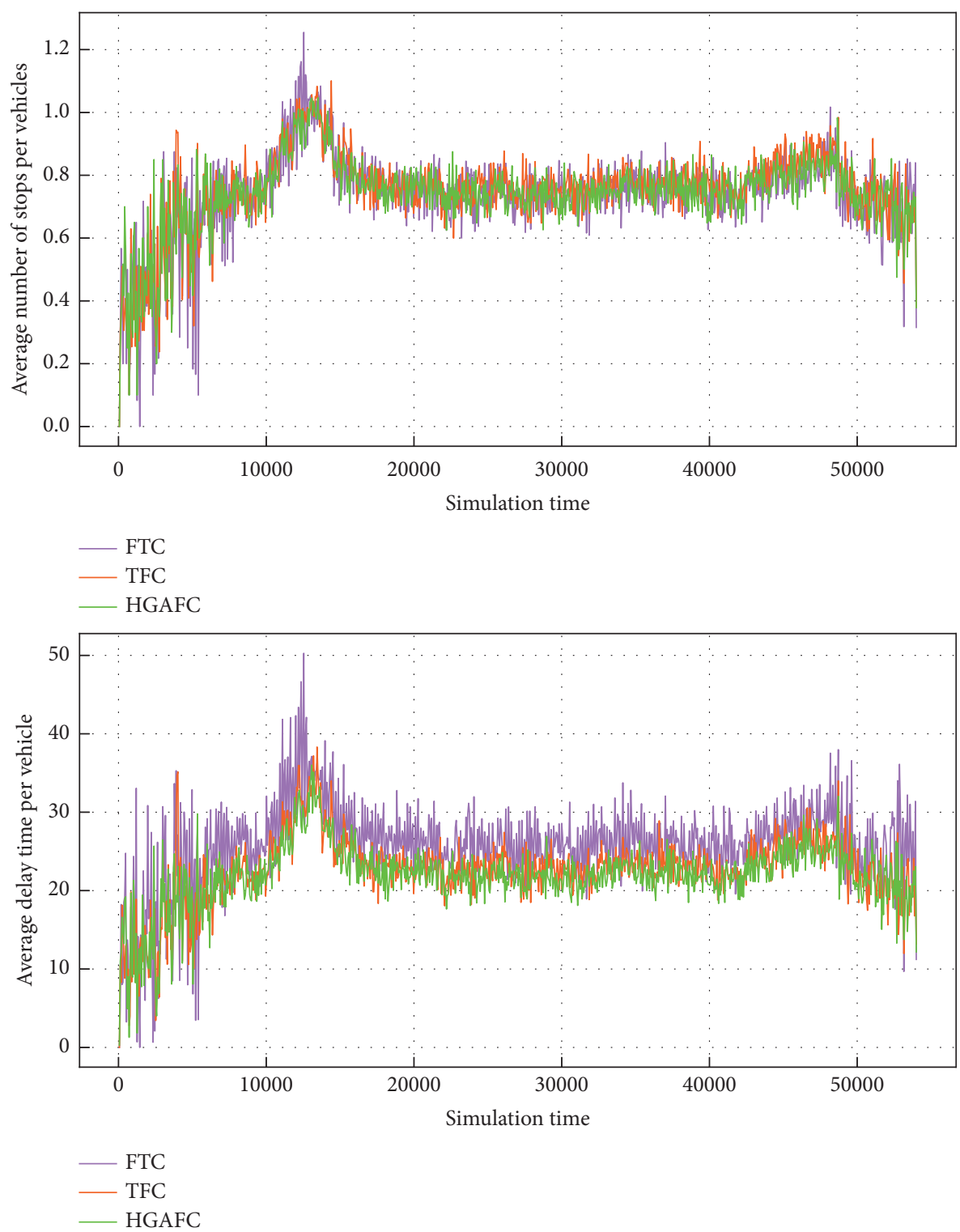

FIgURE 5: Simulation result.

(2) The control algorithm can effectively reduce the average vehicle delay and queue length during peak hours. The reason is that when the traffic flow at the intersection is large, the queuing vehicles cannot get through the parking line in the shortest green time, and the queuing phenomenon begins to appear. The queuing length begins to affect the decision process of the fuzzy controller, and the entrance lane with long queuing begins to obtain the green light extension time, which fully reflects the flexibility and superiority of fuzzy control over timing control.

(3) In the morning rush hour, the optimization effect of fuzzy control on the intersection is weaker than in another period, but it is still better than Fixed-time control. The reason is that when there is more traffic flow at the intersection, the queue length of each approach is longer. In this case, the queue length and phase duration are the main influencing factors of fuzzy control, but the phase duration occupies the dominant position.

(4) Figures 5 and 6 show the changes of critical parameters in the fuzzy control system under different real traffic conditions. In the simulation model, there are 2911 phases in 54000 time steps, i.e., about 727 cycles. The average cycle length of each cycle is 74 seconds, of which 152 cycles change, accounting for 

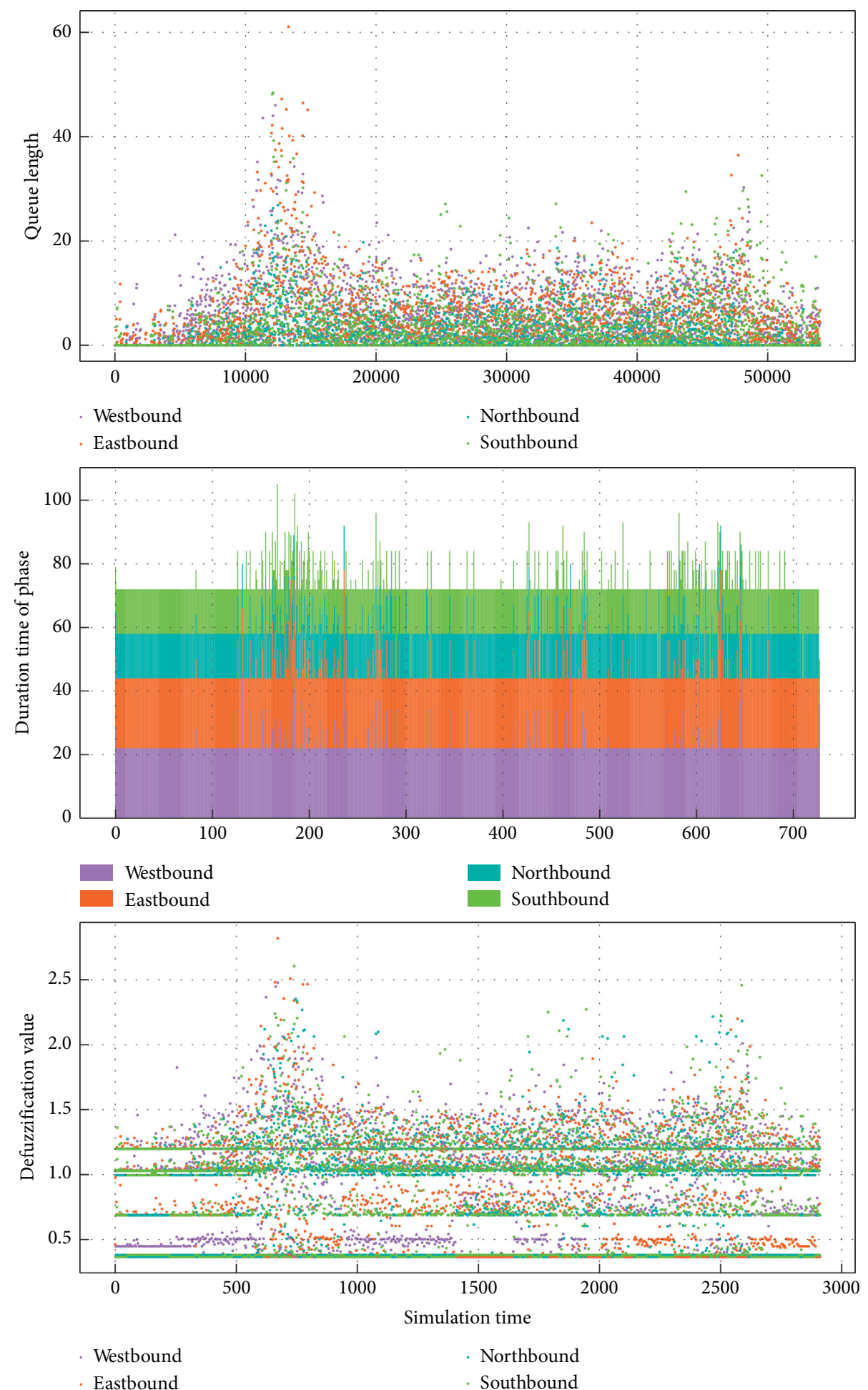

Figure 6: Parameter variation under HGA fuzzy control algorithm.

$20.91 \%$ of the total cycles. It can be seen from the simulation results that the larger the traffic volume at the intersection, the more significant the signal cycle change, which shows that the signal control strategy generated by the algorithm can effectively adapt to the real-time evolution of intersection traffic flow.
Among the 727 cycles, the maximum cycle length is about 100 seconds, and the minimum is 72 seconds. Each phase's maximum green light time is also within a reasonable range, and there are no extreme cases, indicating that the genetic fuzzy control algorithm has good reliability. 

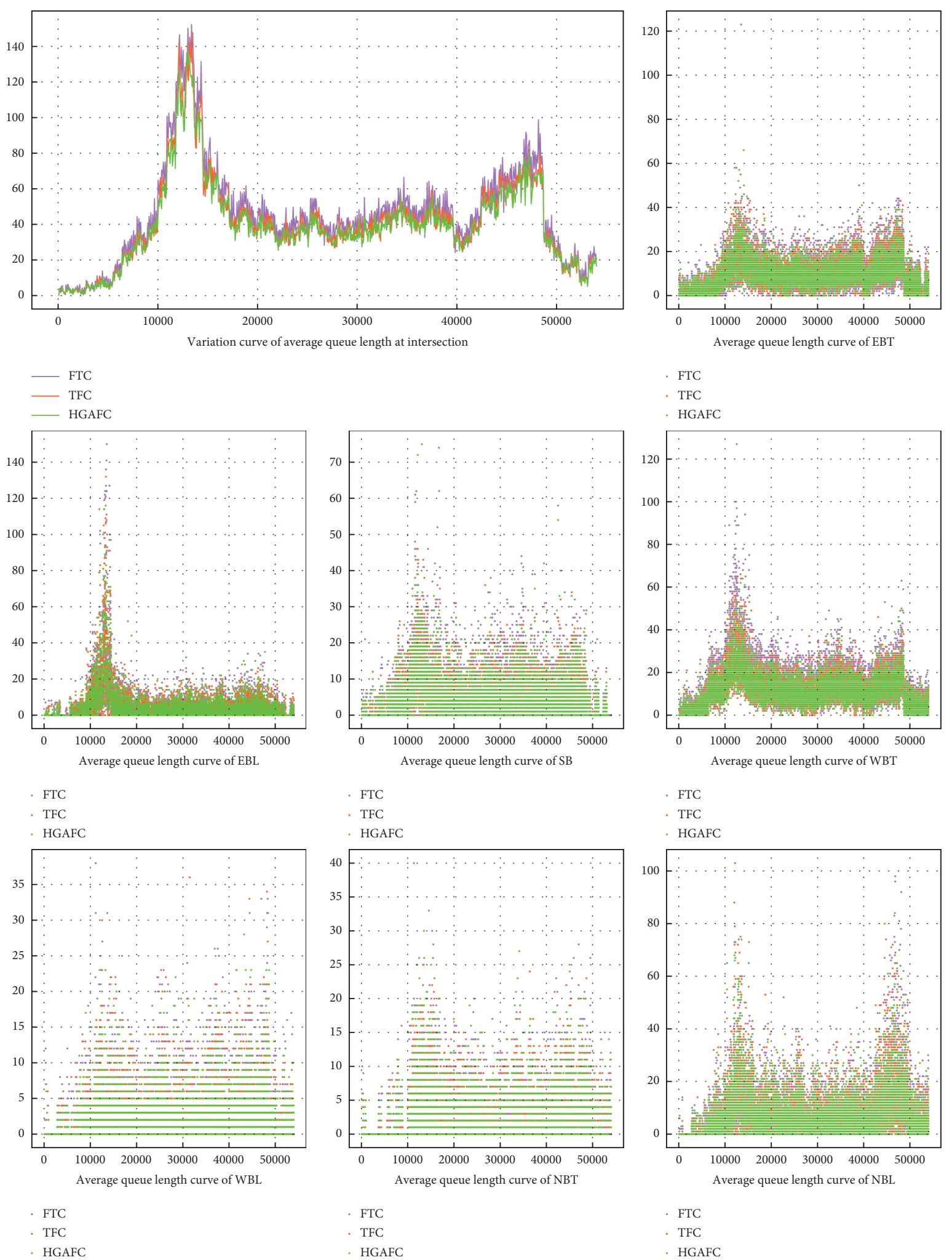

Figure 7: Average queue length curve of different approaches.

\section{Conclusion}

In this study, a self-adaptive two-stage fuzzy controller is proposed, which considers multiple modes of transportation: motor, non-motor, and pedestrian. In order to simulate the driving behavior characteristics and real-time traffic flow fluctuation characteristics of vehicles in the real environment, the simulation parameters of the VISSIM simulation platform are calibrated through the intersection vehicle high-precision trajectory data, and the vehicle traffic 
information extracted based on the trajectory data is used as the input of the simulation platform. Then, its control efficiency and reliability are evaluated based on the microsimulation platform. Results showed that the average delay time per vehicle is reduced by $14.59 \%$, while the average number of stops per vehicle is reduced by $0.71 \%$ compared with the traditional control method. In addition, by analyzing the effectiveness of signal control strategy under different traffic conditions, it is found that the fuzzy controller can only achieve a good control effect under medium saturation or unbalanced traffic flow but has a poor control effect under low or high saturation. This paper researched the optimization question of traffic signal timing in natural traffic conditions, but the optimization module's timeliness was not, so it is best to introduce the traffic flow prediction module in future research.

\section{Data Availability}

Data used to support the findings of this study are available from the corresponding author upon request.

\section{Conflicts of Interest}

The authors declare that there are no conflicts of interest regarding the publication of this study.

\section{Acknowledgments}

This study was supported by project National Key R\&D Program of China (No. 2019YFF0301403).

\section{References}

[1] Y. Yang, Z. Z. Yuan, D. Y. Sun, and X. L. Wen, “Analysis of the factors influencing highway crash risk in different regional types based on improved apriori algorithm," Advances in Transportation Studies, vol. 49, pp. 165-178, 2019.

[2] S. Feng, Z. Li, and Y. Ci, "Risk factors affecting fatal bus accident severity: their impact on different types of bus drivers," Accident Analysis \& Prevention, vol. 86, pp. 29-39, 2016.

[3] R. Aldred, "Cycling near misses: their frequency, impact, and prevention," Transportation Research Part A: Policy and Practice, vol. 90, pp. 69-83, 2016.

[4] Y. Yang, Z. Z. Yuan, J. Y. Li, and Y. H. Wang, "Multi-mode public transit OD prediction and scheduling model," Advances in Transportation Studies, vol. 3, 2018.

[5] M. Drapalyuk, S. Dorokhin, and A. Artemov, "Estimation of efficiency of different traffic management methods in isolated area," Transportation Research Procedia, vol. 50, pp. 106-112, 2020.

[6] Y. Yang, Z. Yuan, J. Chen, and M. Guo, "Assessment of osculating value method based on entropy weight to transportation energy conservation and emission reduction," Environmental Engineering \& Management Journal (EEMJ), vol. 16, no. 10, 2017.

[7] T. Litman, Smart Transportation Emission Reduction Strategies, Victoria Transport Policy Institute, Victoria, Canada, 2017.

[8] W. Li, Z. Pu, and Y. Li, "How does ridesplitting reduce emissions from ridesourcing? A spatiotemporal analysis in
Chengdu, China," Transportation Research Part D: Transport and Environment, vol. 95, Article ID 102885, 2021.

[9] W. Li, S. Chen, J. Dong, and J. Wu, "Exploring the spatial variations of transfer distances between dockless bike-sharing systems and metros," Journal of Transport Geography, vol. 92, Article ID 103032, 2021.

[10] Beijing Transportation Research Center, Beijing Transport Development Annual Report, Beijing Transportation Research Center, Beijing, China, 2021, in Chinese.

[11] W. Li, Z. Pu, and Y. Li, "Characterization of ridesplitting based on observed data: a case study of Chengdu, China," Transportation Research Part C: Emerging Technologies, vol. 100, pp. 330-353, 2019.

[12] K. Tang, M. Boltze, H. Nakamura, and T. Zong, Global Practices on Road Traffic Signal Control: Fixed-Time Control at Isolated Intersections, Elsevier, Amsterdam, Netherland, 2019.

[13] Y. Wenchen, L. Zhang, Z. He, and Y. Yan, "Adaptive twostage fuzzy logic controllers for urban traffic signals at isolated intersections," in Proceedings of the 2012 International Conference on Artificial Intelligence, La Palma, Canary Islands, April 2012.

[14] N. H. Gartner, "A demand-responsive strategy for traffic signal control," in Proceedings of the 62nd Annual Meeting of the Transportation Research Board, pp. 75-81, Transportation Research Board, Washington, DC, USA, January 1983.

[15] F. Lin and D. J. Cooke, "Potential performance characteristics of adaptive control at individual intersections," in Proceedings of the 65th annual meeting of the Transportation, pp. 30-33, Transportation Research Board, Washington, DC, USA, January 1966.

[16] P. Kronborg and F. Davidson, "MOVA and LHOVRA: traffic signal control for isolated intersections," Traffic Engineering and Control, vol. 34, pp. 195-200, 1993.

[17] F. Lin and S. Vijayakumar, "Adaptive signal control at isolated intersections," ASCE Transportation Journal, vol. 114, no. 5, 1988.

[18] C. P. Pappis and E. H. Mamdani, “A fuzzy logic controller for a traffic junction," IEEE Transactions on Systems, Man, and Cybernetics, vol. 7, no. 10, pp. 707-717, 2002.

[19] R. Hoyer and U. Jumar, "An advanced fuzzy controller for traffic lights," Annual Review in Automatic Programming, vol. 19, pp. 67-72, 1994.

[20] M. B. Trabia, M. S. Kaseko, and M. Ande, "A two-stage fuzzy logic controller for traffic signals," Transportation Research Part C: Emerging Technologies, vol. 7, no. 6, pp. 353-367, 1999.

[21] Y. S. Murat and E. Gedizlioglu, "A fuzzy logic multi-phased signal control model for isolated junctions," Transportation Research Part C: Emerging Technologies, vol. 13, no. 1, pp. 19-36, 2005.

[22] S. M. Rahman and N. T. Ratrout, "Review of the fuzzy logic based approach in traffic signal control: prospects in Saudi arabia," Journal of Transportation Systems Engineering and Information Technology, vol. 9, no. 5, pp. 58-70, 2009.

[23] B. M. Nair and J. Cai, "A fuzzy logic controller for isolated signalized intersection with traffic abnormality considered," IEEE Intelligent Vehicles Symposium, pp. 1229-1233, 2007.

[24] J. J. Henry, J. L. Farges, and J. L. Gallego, "Neuro-fuzzy techniques for traffic control," Control Engineering Practice, vol. 6, no. 6, pp. 755-761, 1998.

[25] E. Bingham, "Reinforcement learning in neurofuzzy traffic signal control," European Journal of Operational Research, vol. 131, no. 2, pp. 232-241, 2001.

[26] N. Rida, M. Ouadoud, and A. Hasbi, "Ant colony optimization for real time traffic lights control on a single 
intersection," International Journal of Interactive Mobile Technologies (iJIM), vol. 14, no. 2, pp. 196-214, 2020.

[27] D. Xu, J. Fang, and S. Shao, "A fuzzy controller of traffic system and ITS neural network implementation," Information and Control, vol. 21, pp. 74-79, 1992.

[28] S. Katoch, S. S. Chauhan, and V. Kumar, "A review on genetic algorithm: past, present, and future," Multimedia Tools and Applications, vol. 80, no. 5, pp. 8091-8126, 2021.

[29] J. Liu and X. Zuo, "Research on fuzzy control and optimization for traffic lights at single intersection," Journal of System Simulation, vol. 32, pp. 2401-2408, 2020.

[30] N. P. Shahsavari, H. Asadi, and M. P. Kheradmand, "Fuzzy multiobjective traffic light signal optimization," Journal of Applied Mathematics, vol. 2013, Article ID 249726, 7 pages, 2013.

[31] D. Yu, X. Tian, X. Xing, and S. Gao, "Signal timing optimization based on fuzzy compromise programming for isolated signalized intersection," Mathematical Problems in Engineering, vol. 2016, Article ID 1682394, 12 pages, 2016.

[32] Y. Yang, Z. Yuan, and X. Fu, "Optimization model of taxi fleet size based on GPS tracking data," Sustainability, vol. 11, no. 3, 2019.

[33] M. Z. Wang, Research on the Adjustment of Signal Timing Based on High-Precision Trajectory Data: Taking the Intersection of Beier and Chongzhi Road in Shenzhen as an Example, Beijing Jiaotong University, Beijing, China, 2021.

[34] N. Siddique and H. Adeli, Computational Intelligence: Synergies of Fuzzy Logic, Neural Networks and Evolutionary Computing, John Wiley \& Sons, Hoboken, NJ, USA, 2013.

[35] C. Urrea, J. Kern, and J. Alvarado, "Design and evaluation of a new fuzzy control algorithm applied to a manipulator robot," Applied Sciences, vol. 10, no. 21, Article ID 7482, 2020.

[36] Y. Nie, X. Wu, J. F. Dillenburg, and P. C. Nelson, "Reliable route guidance: a case study from Chicago," Transportation Research Part A: Policy and Practice, vol. 46, no. 2, pp. 403-419, 2012.

[37] B. Park and J. D. Schneeberger, "Microscopic simulation model calibration and validation: case study of vissim simulation model for a coordinated actuated signal system," Transportation Research Record: Journal of the Transportation Research Board, vol. 1856, no. 1, pp. 185-192, 2003.

[38] J. Hourdakis, P. G. Michalopoulos, and J. Kottommannil, "Practical procedure for calibrating microscopic traffic simulation models," Transportation Research Record: Journal of the Transportation Research Board, vol. 1852, no. 1, pp. 130-139, 2003.

[39] L. Chu, H. X. Liu, J. S. Oh, and W. Recker, "A calibration procedure for microscopic traffic simulation," in Proceedings of the 2003 IEEE International Conference on Intelligent Transportation Systems, vol. 2, Shanghai, China, October 2003.

[40] M. Hosamo, "A study of the source traffic generator using Poisson distribution for ABR service," Modelling and Simulation in Engineering, vol. 2012, Article ID 408395, 6 pages, 2012 . 